Tatjana Č. Đurin ${ }^{1}$

DOI: https://doi.org/10.46630/phm.12.2020.14

Université de Novi Sad

Article de recherche

Faculté de philosophie ${ }^{2}$

УДК 811.133.1'255:811.163.41

$741.5(44)=163.41$

Département d'études romanes

Reçu : le 21/2/2020

Nataša N. Popović

Université de Novi Sad

Faculté de philosophie

Département d'études romanes

\title{
DEUX AUBERGES DU LOINTAIN : DEUX TRADUCTIONS SERBES DE LA BANDE DESSINÉE ASTÉRIX EN CORSE
}

Dans le présent travail, nous nous intéressons à deux traductions serbes du $20^{\mathrm{e}}$ album de la série Astérix, intitulé Astérix en Corse. Nous faisons une analyse comparative des stratégies de traduction auxquelles les traducteurs Đorđe Dimitrijević et Ivan Klajn recourent, ainsi que des solutions qu'ils proposent. Nous nous focalisons plus particulièrement sur les dimensions humoristiques des deux traductions afin de voir si les traducteurs réussissent à préserver l'effet humoristique du texte source et si leurs traductions sont bien adaptées aux exigences du public cible. Notre analyse, fondée sur l'approche traductologique d'Antoine Berman (1999), porte sur différentes sources du comique : interactions verbo-iconiques, jeux de mots, diversité linguistique, langage familier, références culturelles et noms propres. Même si nous pouvons constater que les deux traducteurs se servent de différents procédés, ils réussissent tout de même à transmettre le comique d'Astérix, de sorte que la bande dessinée, dans toute sa verbo-iconicité, continue à vivre dans un autre espace linguo-culturel, en faisant rire un autre public.

Mots-clés : traduction, bande dessinée, Astérix en Corse, humour, le français, le serbe

\section{Introduction}

L'année dernière Astérix a fêté ses 60 ans. La première planche des Aventures d'Astérix le Gaulois, créée par René Goscinny (scénariste) et

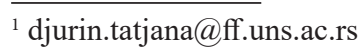

${ }^{2}$ Cette recherche contribue au projet scientifique $\mathrm{N}^{\circ} 178002$ (Les langues et les cultures dans le temps et dans l'espace) financé par le ministère de l'Éducation, de la Science et du Développement technologique de la République de Serbie. 
Albert Uderzo (dessinateur), a paru dans le premier numéro de Pilote, grand hebdomadaire français destiné à la jeunesse, en 1959. Deux années plus tard, en 1961, Astérix le Gaulois, le premier album de la série de la bande dessinée Astérix, a été publié. La série a connu un succès éditorial exceptionnel, unique dans le domaine de la bande dessinée, avec plus de 370 millions d'exemplaires vendus en 60 ans dans le monde entier. Il a été traduit en 112 langues et dialectes (CROS 2011 : 19). La première traduction serbo-croate d'Astérix a paru en 1966, tandis que la version serbe a été publiée en 1995 (CROS 2011 :20).

En Serbie, la série Astérix est un élément incontournable de la culture générale, surtout grâce à ses traducteurs, Đorđe Dimitrijević et Ivan Klajn. Outre Astérix, Đorđe Dimitrijević a également traduit de nombreuses autres bandes dessinées, telles que Gaston Lagaffe, Lucky Luke ou Iznogoud. Ivan Klajn, académicien et linguiste, a longtemps collaboré avec le magazine «Politikin zabavnik » pour lequel il a traduit, pendant des années, des bandes dessinées françaises : Astérix, Iznogoud, Lucky Luke et Tintin. (STEVANOVIĆ 2010). ${ }^{3}$

La spécificité de la bande dessinée réside dans l'interaction entre le code visuel et le code graphique, éléments indissociables et complémentaires. Par conséquent, la tâche du traducteur consiste à décoder l'image et le texte, sans perdre de vue les attentes du public cible. D’après A. Rollo, « la traduction de la bande dessinée partage plusieurs traits avec la traduction audiovisuelle, en premier lieu pour l'intégration des codes verbaux et non verbaux dans un langage unitaire, de manière à créer une unité multidimensionnelle, où la composante visuelle conditionne le transfert de l'élément verbal » (ROLLO 2017 : 187). Dans la BD, l'image représente « un élément constitutif du texte, qui participe à la création du sens et qui devient au besoin une source d'humour » (ROLLO 2017 : 188). Cependant, « les traductologues qui s'occupent des difficultés traductives inhérentes à une bande dessinée privilégient souvent le code verbal strictu sensu - jeux de mots, assonances, etc. - comme source principale des effets comiques, en négligeant d'autres facteurs, tels que l'ironie, les stéréotypes sociaux, les références explicites à la culture d'origine »(ROLLO 2017 : 188). Pourtant, l'humour est toujours en lien étroit avec les aspects socioculturels spécifiques d'une communauté (traditions, mœurs, croyances, etc.).

\footnotetext{
${ }^{3}$ Il faut souligner le fait que, lorsqu'on traduit les albums d'Astérix dans une langue étrangère, les versions traduites sont toujours " relues et retraduites en français par des vérificateurs, parfaitement bilingues [...], qui doivent contrôler que le registre de vocabulaire qui a été choisi correspond bien à celui de l'univers d'Astérix et que les marqueurs humoristiques sont compréhensibles dans les différents pays. La traduction ne doit jamais être une simple transcription ou une transposition d'un texte » (TOUILLIER-FEYRABEND $1998: 375$ ).
} 
La série des albums d'Astérix est basée sur un humour « très français » et comporte de nombreux éléments de la culture et de l'histoire françaises. L'un des principes de base de ces albums est une subtile (con)fusion temporelle entre le monde gallo-romain et celui de la France contemporaine (KAUFMANN 1998 : 330). En d'autres termes, Astérix neutralise la distance temporelle et « identifie les Gaulois aux Français. Deux récits, l'un héroïque et historique, l'autre parodique et ouvertement fictif, se déploient alors en parallèle » (MAGUET 1998 : 317). De même, la série regorge de stéréotypes et de particularismes locaux et nationaux, que les lecteurs issus de cultures très éloignées ne peuvent pas identifier (PALMA 2006 : 901). Lors de la transmission des dimensions humoristique et culturelle dans la langue cible, les traducteurs des albums d'Astérix peuvent rencontrer de nombreuses difficultés culturelles (culturèmes, stéréotypes) et linguistiques (noms propres, dialectes, jeux de mots, expressions figées, etc.). L'action de nombreux albums se déroule hors du village. Par conséquent, « outre la caricature habituelle des Romains et la sympathique satire des mœurs gauloises (en réalité françaises), la représentation des peuples voisins fournit un réservoir inépuisable de stéréotypes nationaux » (MEYER $2011: 169$ ).

\section{Analyse du corpus}

Notre analyse porte sur deux traductions serbes du $20^{\mathrm{e}}$ album de la série Astérix, intitulé Astérix en Corse, publié en 1973. Nous ferons une analyse comparative des stratégies de traduction auxquelles les traducteurs Đorđe Dimitrijević et Ivan Klajn recourent, ainsi que des solutions qu'ils proposent. L'approche traductologique qui sera adoptée est celle d'Antoine Berman qui oppose la traduction ethnocentrique, « qui ramène tout à sa propre culture, à ses normes et valeurs, et considère ce qui est situé en dehors de celle-ci - l'Étranger - comme négatif ou tout juste bon à être annexé, adopté, pour accroître la richesse de cette culture » (BERMAN 1999 : 29), à la traduction éthique, qui reconnaît et reçoit " l'Autre en tant qu'Autre », qui accueille "l'Autre, l'Étranger, au lieu de le repousser ou de chercher à le dominer » (BERMAN 1999 : 74-75).

\subsection{Les interactions verbo-iconiques et les jeux de mots}

Puisque la caractéristique principale de la bande dessinée est sa nature verbo-iconique (POPOVIĆ, NIKOLIĆ 2017 : 358), le traducteur doit, lui aussi, tenir compte de cette interaction de l'image et du texte. Les deux traducteurs serbes transposent adéquatement les relations entre le dessin et le 
mot mais, dans certains cas, ces relations sont introduites dans la traduction là où elles n'existent pas dans l'original.
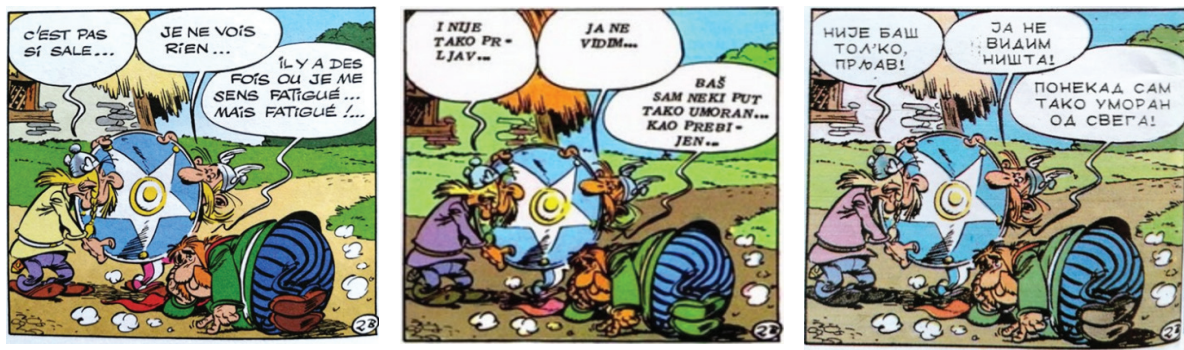

La résignation d'Abraracourcix $(\mathrm{AC}, 6)$ est traduite littéralement par Klajn $(K, 8)$, qui reproduit ainsi l'effet comique du texte source. Dimitrijević $(D, 5)$ y ajoute un nouvel effet comique basé sur le rapport entre l'image et le texte. Il propose une traduction libre (kao prebijen), jouant avec la polysémie du verbe prebiti, qui signifie 'casser' mais aussi 'battre, tourmenter physiquement et moralement' (RMS 2007 : 1001).

De même, dans un autre exemple (AC, 13 ; D, 12 ; K, 15), Dimitrijević renforce le lien entre le dessin et le texte :
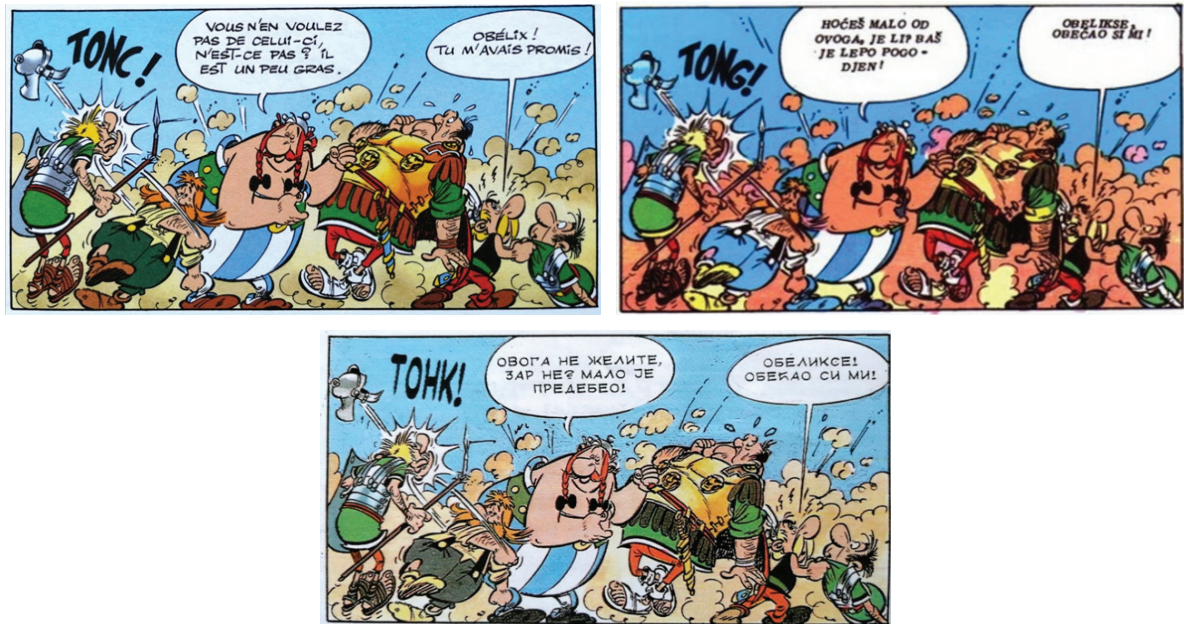

La traduction de Klajn est littérale adéquate et, comme le texte source, fait référence au dialogue dans lequel Astérix exige d'Obélix de laisser les plus beaux Romains pour les invités. Obélix lui répond : "Oui, mais tu sais, les Romains c'est comme les huîtres : les petits sont souvent les meilleurs ! » (AC, 10). La traduction de Dimitrijević est, par contre, libre, basée sur la polysémie du participe passé pogođen, qui signifie 'frappé, atteint' mais aussi 
'réussi, bien fait (comme il faut)'.

Les jeux de mots, ancrés dans la langue source et, par la suite, dans la culture source, représentent toujours un grand défi lancé aux traducteurs ${ }^{4}$.

Oh! Regarde! Des sangliers domestiques !/ Non. Ce sont des cochons sauvages. $(\mathrm{AC}, 25)$

Oh, gledajte! Domaći prasići!/ Ne, to su divlje svinje. (D, 24)

Ox! Гледај! Припитомљени дивљи вепрови!/ Не, то су подивљале домаће свиње! (К, 27)

Le comique repose sur l'oxymore qui n'a pas été transmis par Dimitrijević, la difficulté étant que le terme serbe désignant le sanglier contient déjà l'adjectif 'sauvage' : divlja svinja, divlji vepar. Tandis que Dimitrijević propose une sous-traduction, Klajn, en se servant du procédé d'ajout, réussit à traduire l'oxymore : припитомљени дивљи вепрови 'des sangliers apprivoisés'/ подивљале домаће свиње 'des cochons devenus sauvages'.

\subsection{La diversité linguistique}

Les mots et les phrases latins insérés dans le texte français engendrent le comique puisqu'ils contribuent, avec les dessins (en général des caricatures), à la création de la couleur locale :

mes caligae me font souffrir (AC, 22$)^{5}$

žulje me kalige $(\mathrm{D}, 21)$

jep ме моје caligae грозно жуље $(\mathrm{K}, 24)$

En utilisant le procédé de transcription, Dimitrijević transmet l'effet comique, mais Klajn, qui opte pour l'emprunt direct, crée un nouvel effet comique dont la source est l'invraisemblable : le mot latin dans le texte cyrillique.

Les proverbes et les citations latins sont toujours transposés en serbe, mais différemment :

E"a'e humanum est! (AC, 19)

Errare humanum est! $(\mathrm{D}, 18)$

E"a'e humanum est! (K, 21)

En transposant les proverbes prononcés par Baba, Klajn se sert d'emprunt direct et transpose également l'image visuelle de sa prononciation fautive, tandis que Dimitrijević opte pour le procédé d'explicitation et utilise les proverbes originaux pour faciliter la compréhension.

Le trouble du langage du petit Gaulois, évident dans la fameuse citation

\footnotetext{
${ }^{4}$ Sur la traduction des jeux de mots, voir HENRY 2003.

${ }^{5}$ Dans tous les exemples cités, c'est nous qui soulignons.
} 
de César, «Alea zacta eft ! » $(\mathrm{AC}, 5) /$ « Napreed ! » $(\mathrm{D}, 4) /$ «Alea acta eft ! » $(\mathrm{K}, 7)$, est transposé par Klajn. Dimitrijević, par contre, propose une traduction libre mais adéquate (En avant!), bien adaptée au dessin et au contexte.

La citation de Virgile (Georgicon, II, 490), " Felix qui potuit rerum cognoscere causas ${ }^{6}$, comme on dit vulgairement » (AC, 22), prononcée par l'un des pirates, dans la version de Klajn a été traduite par l'emprunt direct: « Felix qui potuit rerum cognoscere causas, што би рек'о прост народ » (K, 24). Dimitrijević a choisi le procédé de substitution : il remplace la citation de Virgile par un proverbe serbe et change le point de vue : "Znate kako se naučno kaže: koga su ujedale zmije, boji se i guštera ${ }^{7} »(\mathrm{D}, 21)$. Un effet comique est tout de même transmis.

Les dialectes français, en tant que traits distinctifs des personnages dans Astérix en Corse, représentent l'un des mécanismes linguistiques de l'humour. Parmi les invités qui arrivent dans le village pour fêter l'anniversaire de Gergovie, le lecteur reconnait une famille de l'Auvergne. Les auteurs utilisent l'un des traits caractéristiques de l'auvergnat, le passage de $\mathrm{S}$ en [J] et [3] devant I et $U$ en position intervocalique, le trait linguistique poussé à l'extrême dans la bande dessinée. Puisque le lecteur cible ne connaît pas les caractéristiques de l'auvergnat, les traducteurs serbes ont été obligés de remplacer cette référence linguo-culturelle par un trouble du langage (Klajn le fait systématiquement, tandis que Dimitrijević ne le fait que sporadiquement), ou bien de l'omettre complètement (le plus souvent dans la version de Dimitrijević) :

Chalut ! Je chuis chinchèrement ravi de te revoir! $(\mathrm{AC}, 9)$

Ždravo društvo! Drago mi je što vas vidim! (D, 8)

Ждраво! Ишкрено шам шрећан што ше опет шаштајемо! (K, 11)

Un des invités, Labeldecadix, l'aubergiste de Massilia, parle marseillais, avec ses traits caractéristiques poussés à l'extrême (E indistinct, diérèse de certaines diphtongues, prononciation particulière des voyelles nasales finales, usages fréquents des impératifs des verbes pour attirer l'attention). Dans les dialogues d'Alambix (auvergnat) et Labeldecadix (marseillais), les traducteurs serbes ont été forcés de se débrouiller. Dans la version de Dimitrijević, l'auvergnat est remplacé par un trouble du langage, tandis que le marseillais est remplacé par le dialecte du sud de la Serbie. L'accent est mis sur le lexique (čarlama 'sorte de danse' mais aussi 'tricherie, manipulation', krdža 'tabac de mauvaise qualité', popara 'panade', bre, interjection utilisée dans toutes les situations) et sur les formes et l'emploi particulier des pronoms personnels $(g i, s i)$. Klajn, par contre, joue avec la prononciation (свечано-ишпеченошвечано; свечано-сечено-швечано-шечено), proposant une traduction

\footnotetext{
${ }^{6}$ Heureux qui a pu pénétrer la raison des choses.

${ }^{7}$ Vous savez, comme on dit scientifiquement : qui a été mordu par les serpents, craint les lézards.
} 
moins expressive mais qui, tout de même, transmet un effet comique produit par la diversité linguistique du texte source :

Vé ! C'est un peu bieng organisée, cette faîte !/ Chette quoi ?/ Cette faîte ! Effeu-ê-té-eu. Cette faîte !/ Ah ! Chette fête !/ Vouaye. Cette sôterie si vous préférez. $(\mathrm{AC}, 11)$

Jes, jes! Ima da gi udarimo čarlamu!/ Šta šemo?.../ Čarlamu, bre!... Odakle si kad ne znaš šta je to?.../ Ah! Šarlamu!/ Pa, jes! El poparu da gi skuvamo, ako ti je to lepše! (D, 10)

Лепо је овде! Весело и свечано!/ Шта је ишпечено?/ Кажем! Весело и свечано!/ А! Швечано!/ Можете и на овај излет ако више волите! $(\mathrm{K}, 13)$

L'emploi des parlers locaux le plus intéressant dans Astérix en Corse est celui des parlers monténégrins utilisés par Dimitrijević, plus ou moins systématiquement, pour traduire les propos des personnages d'origine corse. Certaines caractéristiques des parlers monténégrins, qui ont pour base le dialecte chtokavien (de prononciation ijékavienne) de la langue jadis appelée le serbocroate, représentent des traits distinctifs des Corses dans la bande dessinée.

On y voit des exemples de l'emploi des formes atones des pronoms personnels au datif (1/Pl et 2/Pl), ni et vi, au lieu de nam et vam :

Oui, je savais bien qu'ils ne voudraient pas le garder. (AC, 25)

Jes, jes, znao sam da će ni ga brzo vratit! $(\mathrm{D}, 24)$

Да! Знао сам ја да неће хтети да га задрже! (K, 27)

Dans cet exemple, on peut également remarquer l'emploi de l'infinitif (en général plus fréquent qu'en serbe) sans I final (vratit au lieu de vratiti).

La traduction de Dimitrijević présente également des exemples de la palatalisation ijékavienne typique au Monténégro $(\mathrm{D}+\mathrm{J}=[\mathrm{d} z])$, ainsi que ceux de la réduction du groupe vocalique $\mathrm{AO}$ à $\mathrm{A}$ en position finale :

Regarde : c'est pas plus gros qu'une châtaigne, mais ça mange comme si sa sieste en dépendait. (AC, 26)

Viđi ga kaki je, ka vrabac, a jede ka jedna ala! (D, 25)

Погледај га! Шака јада, а ждере као да му је сијеста у питању! $(\mathrm{K}, 28)$

Cette réduction du groupe $\mathrm{AO}$ est souvent présente à la fin de la forme du masculin singulier du participe passé actif des verbes : reka sam au lieu de rekao sam 'j'ai dit'. Ainsi dans la traduction de Dimitrijević :

Acceptes-tu de prendre discrètement des passagers pour la Corse ? Ton prix sera le nôtre. (AC, 18)

Bi li prista da neke uzmeš tajno do Korzike? Za cenu ne pitamo. (D, 17)

Би ли примио, у поверењу, три путника за Корзику? Плаћамо колико тражиш. (K, 20)

La forme de datif, instrumental et locatif des adjectifs qualificatifs, des 
pronoms et des adjectifs numéraux contient souvent le groupe IJE au lieu de I (ovijeh au lieu de ovih). Ce trait typique des parlers monténégrins est aussi présent dans la traduction de Dimitrijević :

Les autres ne sont pas d'ici. (AC, 25)

Oni drugi nisu od našijeh! (D, 24)

Они други нису одавде! (K, 27)

La forme de datif en E, au lieu de I, de certains pronoms personnels se trouve aussi dans la traduction de Dimitrijević :

Je ne me bats pas sous les ordres d'un merle ! $(\mathrm{AC}, 40)$

Mene neće da komanduje svaka šuša! (D, 39)

Jа не примам наредбе од жутокљунца! (K, 42)

À part les mots et les expressions caractéristiques des parlers monténégrins (pašče pour le chien (AC, 25; D, 24) $)^{8}$,junak pour le plus fort $(\mathrm{AC}, 25 ; \mathrm{D}, 24)^{9}$, beda od vojnika pour des minables (AC, $21 ; \mathrm{D}, 20)^{10}$ etc.), dans la traduction de Dimitrijević, on repère l'emploi fréquent de l'aoriste (le passé simple), forme verbale considérée comme littéraire à nuance archaïque dans le serbe standard. En traduisant les commentaires des vieillards qui regardent la bataille d'Aléria (AC, 43 ; D, 42), Dimitrijević utilise l'aoriste là où Goscinny utilise le présent ou le passé composé : obori pour assomme, uze pour a épousé, ujede pour mord, predadoše se pour se rendent. Klajn, par contre, utilise le présent et le parfait qui sont d'usage courant en serbe.

Pourtant, ce ne sont pas seulement les traits linguistiques qui font allusion aux Monténégrins. Il y a aussi une référence culturelle, très subtile mais reconnaissable pour le lecteur cible:

Mais moi, avec mes hommes, je reprends tout ce qu'il y a dans les entrepôts avant le départ du préteur! Jusqu'à présent César n'a pas eu une châtaigne à se mettre sous la dent! $(\mathrm{AC}, 15)$

Ali ja i moji junaci na sablju povratimo sve što pretor uzme, tako da Cezar do sada nije dobio od nas ni orah da zagrize! $(\mathrm{D}, 14)^{11}$

Le mot châtaigne, référence culturelle, a été remplacé par le mot orah 'la noix', allusion aux vers de Petar Petrović Njegoš (1813-1851), poète et prince-évêque du Monténégro, qui parlent de la résistance face aux oppresseurs : Tvrd je orah voćka čudnovata,/ ne slomi ga, al'zube polomi! (NJEGOŠ 1959 : v. 1133-1134) ${ }^{12}$.

\footnotetext{
${ }^{8}$ Klajn: pas $(\mathrm{K}, 27)$.

${ }^{9}$ Klajn: najjači $(\mathrm{K}, 27)$.

${ }^{10}$ Klajn: najgori vojnici $(\mathrm{K}, 23)$.

${ }^{11}$ Klajn: Али ја, са својим људима отимам све из његових складишта пре него што се претор врати! До данас, Цезар с Корзике није добио ни један једини кестен (K, 17).

${ }^{12}$ La noix dure est un fruit extraordinaire, - / tu ne le brises pas, mais contre lui tu casses tes dents ! (NIÉGOCH 1917 : v. 1133-1134).
} 
L'emploi des parlers monténégrins est assez systématique mais il n'est pas excessif. Les parlers monténégrins ont été choisis en tant qu'équivalent fonctionnel pour transmettre certains stéréotypes des Corses qui sont en général identiques aux stéréotypes des Monténégrins : l'honneur, la fierté, la paresse, la vendetta, la résistance, les clans etc. La traduction de Klajn, quoiqu'elle soit plus littérale, plus correcte, est en fait moins expressive, moins comique, mais fidèle à l'original.

\subsection{Le langage familier}

Les termes et les expressions appartenant au registre familier, qui, eux aussi, représentent une source du comique dans les bandes dessinées, sont traduits en serbe au moyen de différents procédés. Il y a des exemples de la traduction littérale adéquate, tel que :

Bien garçons. Nous sommes assez loin des côtes. Allons plumer ces trois pigeons $(\mathrm{AC}, 19)$

Čujte momci, sad smo odmakli od obale. Možemo da očerupamo golubove! (D, 18)

Момци, сад смо доста далеко од обале! Хајде да очерупамо та три голупчета! $(\mathrm{K}, 21)$

L'usage familier du verbe plumer 'voler' existe dans les deux langues.

Dans un autre exemple, Dimitrijević propose une traduction littérale adéquate, tandis que Klajn, en changeant le registre de langue, propose une sous-traduction :

C'est son petit voyou qui a dit à mon gosse que je vendais des poiss... (AC, 6) Onaj njegov mangup je rekao mom švrći da ja prodajem kv... (D, 5)

Његов мали мангуп рекао је мом сину да ја продајем тр... $(\mathrm{K}, 8)$

Le mot gosse, dans la version de Dimitrijević traduit par son équivalent fonctionnel ( $\check{s} v r c ́ a)$, dans la version de Klajn perd son expressivité et son affectivité, étant donné qu'il est traduit par un terme neutre du langage standard, sin 'fils'.

À la différence de Klajn, qui le fait très rarement, Dimitrijević se sert, dans la plupart des cas, du procédé de surtraduction, c'est-à-dire il utilise le langage familier là où il n'est pas utilisé dans le texte source :

Allez jouer ailleurs, les enfants! (AC, 5)

Čistanjac klinci! Igrajte se na drugom mestu! (D, 4)

Децо, играјте се негде другде! (K, 7)

Tandis que Klajn propose une traduction littérale adéquate, Dimitrijević renforce l'effet comique en utilisant deux mots appartenant au registre familier : čistanjac, un néologisme créé d'après le verbe čistiti se 'se nettoyer', qui, dans le langage familier signifie 's'enfuir, dégager', et klinci, enfants. 
Il m'enverra en Gaule... Il paraît qu'il y a là-bas un village où ils sont pires que des Corses... Et eux, ils n'ont que du poisson... / Et pas toujours frais, dit-on. (AC, 28) ... Mogu odmah da se pakujem za Galiju... Kažu da tamo ima neko selo gde su još gori od Korzikanaca!/ ... A od klope samo smrdljiva riba! (D, 27)

... Послаће ме у Галију! Прича се да тамо има једно село где су гори него Корзиканци... А од робе имају само рибу.../ ... и то не баш свежу! (K, 30)

Klajn traduit littéralement, en ajoutant le mot roba 'marchandise', faisant allusion à l'avidité et aux pillages du préteur Suelburnus. Pour traduire la phrase il m'enverra en Gaule, Dimitrijević utilise l'expression familière pakovati se (le plus souvent employée à l'impératif : pakuj se ! 'va t'en ! dégage !'). De même, là où Klajn ajoute le mot roba, Dimitrijević ajoute le mot klopa, qui appartient au registre familier et désigne la nourriture, la bouffe.

En somme, les solutions de Klajn représentent des équivalents fonctionnels qui transmettent la signification et l'effet comique, tels qu'ils sont dans le texte source. Par contre, tout en transposant le sens, par l'emploi constant du langage familier, Dimitrijević, soit emporté par son élan créatif, soit conscient de la nécessité de l'emploi du procédé de compensation (pour compenser de ce qui est omis ailleurs), renforce l'effet comique.

\subsection{Les références culturelles}

Les références culturelles (culturèmes) représentent l'une des plus constantes et des plus riches sources du comique. Les éléments culturels, c'est-à-dire des mots et des syntagmes typiques du mode de vie, de la culture, du développement social et culturel d'une société, désignent une spécificité locale ou historique (POPOVIĆ, NIKOLIĆ 2017 : 362) ${ }^{13}$. Étant donné qu'ils n'ont pas d'équivalents formels dans d'autres langues-cultures, ils nécessitent une approche spéciale lors du processus de traduction (FLORIN 1993 : 123).

Dans Astérix en Corse, les culturèmes, dont la fonction principale est d'engendrer le comique, proviennent de différentes cultures : gauloise, romaine, corse, française.

\subsection{La culture gauloise}

Dans la traduction de Klajn les noms des dieux celtes, Toutatis et Bélénos $(\mathrm{AC}, 5)$, sont transposés $(\mathrm{K}, 7)$, tandis que Dimitrijević remplace ce culturème par le juron serbe, tako im sunca kalajisanog, (littéralement : Par leur soleil étamé !) qui fait référence à l'ancien dieu slave Dabog ${ }^{14}$.

C'est Patologix, notre druide. Il est en train de cueillir le gui. (AC, 24)

\footnotetext{
${ }^{13}$ Voir également BALLARD 2005 : 15 ; FARINA $2011: 474$.

${ }^{14}$ Sur Dabog, voir ĐURIN, JOVANOVIĆ 2019 : 110-111.
} 
To je Travariks, naš druid. Došao je da bere dren. (D, 23)

То је Панегирикс, наш друид! Дошао је да бере имелу! $(\mathrm{K}, 26)$

Le gui sacré, coupé par les druides le sixième jour de l'année celtique, symbolisait la vie perpétuelle. On lui attribuait des vertus médicinales, étant donné qu'il guérissait le corps et purifiaitl'âme(CHEVALIER, GHEERBRANT 1982 : 492). La traduction de Klajn est littérale (imela), mais elle ne transpose pas toutes ces connotations culturelles du gui, qui, dans la culture serbe est en général perçu comme une plante parasite. Par contre, Dimitrijević, qui utilise la stratégie de naturalisation, réussit à traduire le culturème. Il remplace le gui par dren 'cornouiller mâle', une plante considérée comme sacrée dans la culture serbe. De la même façon que le gui, le cornouiller est rituellement cueilli une fois par an, la veille de Noël. Le cornouiller est un symbole de bonne santé, d'où le dicton populaire zdrav kao dren, qui signifie littéralement 'sain comme le cornouiller'.

\subsection{La culture romaine}

Par Jupiter ! Ça grouille de cochons par ici !/ Une voie romaine ! Qu'on me donne une voie romaine ! (AC, 34)

Jupitera mu! Ala je ovo neki gustiš!/ Budalo jedna! Vidiš da je gusto na sve strane! (D, 33)

Јупитера му! Овде све врви од свиња!/ Ах, римски друм! Дајте ми наш лепи римски друм! $(\mathrm{K}, 36)$

Le nom du dieu suprême de la mythologie romaine a été conservé dans les deux traductions, mais Dimitrijević omet un autre culturème. La voie romaine, symbole de la puissance de Rome, de sa civilisation et sa société organisées, n'est pas transposée dans la version de Dimitrijević qui propose une traduction libre, un jeu de mots avec l'adjectif gust 'touffu' et le substantif gustiš 'fourré'. Puisque le terme est connu dans la culture cible, dont le territoire faisait partie de l'Empire romain, Klajn transmet le culturème, en assurant la compréhension par l'ajout de l'adjectif lepi 'beau'.

\subsection{La culture corse}

Astérix en Corse présente de nombreuses allusions à la culture corse, surtout aux stéréotypes, tels que la paresse (la sieste pratiquée presque religieusement), l'honneur, la vendetta, les sangliers et les châtaignes traditionnellement utilisés dans l'alimentation, le maquis, et, bien sûr, Napoléon Bonaparte.

Après la sieste ! (AC, 12)

Posle dremke... (D, 11)

Док одремам! Сијеста! (K, 14) 
Emprunté à l'espagnol, le mot sieste désigne le temps de repos, avec ou sans sommeil, qui se prend après le repas de midi, surtout dans les pays chauds (TLFi 2004). Klajn le traduit littéralement, par le même mot, emprunté, en serbe aussi, à l'espagnol. En outre, pour assurer la compréhension, il ajoute une explicitation: Dok odremam 'dès que je termine mon petit somme'. Dans la traduction de Dimitrijević, le culturème est omis mais le comique est tout de même assuré par l'emploi du terme familier dremka 'roupillon'.

Vienivienivieni./ Aïe aïeaïeaïe, vous pouvez embarquer. (AC, 19)

Idimidođimi!/ Aha, aha, za vas je slobodno! Uđite! (D, 18)

Воларее, о, о!/ Кантареее, о, о, o, o! (K, 21)

Le mot de passe demandé pour pouvoir s'embarquer pour la Corse, ainsi que la réponse, font allusions aux chansons du chanteur corse Tino Rossi (Vieni vieni et La danse à la mode). Dimitrijević omet les culturèmes et remplace le mot de passe par une expression familière serbe Idi mi, dođi mi 'patati patata' (RMS 2007 : 315). Klajn se sert, lui aussi, de procédé de substitution mais il remplace les culturèmes corses par des culturèmes italiens, plus connus dans la culture cible : les paroles de la chanson de Domenico Modugno et Franco Migliacci, Nel blu dipinto di blu, plus connue sous le titre Volare.

Je crois que les Romains vont faire une cueillette de châtaignes... (AC, 38)

Čini mi se da Rimljani danas imaju branje bostana! (D, 37)

Мислим да ће Римљани извући добре батине! (K, 40)

Récoltée et cultivée en Corse, la châtaigne avait été considérée comme une source de la paresse, de l'ignorance et de la misère, étant donné que les personnes qui se nourrissaient en cueillant la châtaigne ne cultivaient rien, ne travaillaient pas vraiment. Dans cet exemple, le culturème se combine avec le registre du langage populaire où le mot châtaigne désigne un coup de poing. Klajn omet le culturème et propose une explicitation : les Romains recevront de beaux coups de poing. Dimitrijević, lui aussi, omet le culturème et propose une traduction partielle, en remplaçant la cueillette de châtaigne par la cueillette de pastèques, d'après l'expression serbe, appartenant elle aussi au registre familier, obrati bostan 'périr, souffrir'.

Le maquis $(\mathrm{AC}, 30)$, une formation végétale caractéristique des régions méditerranéennes, représente un culturème polyvalent : en Corse, le lieu où les gens se réfugiaient pour échapper à la justice ou à la vendetta ; le lieu où des groupes de résistants s'organisaient pour lutter contre les nazis durant la Seconde Guerre mondiale ; en Corse, le lieu où de nombreux touristes se perdent. Klajn propose une traduction partielle, čestar (K, 32), en transposant seulement l'aspect botanique du terme maquis. Dimitrijević, par contre, choisit le mot šuma 'forêt' (D, 29), qui représente un équivalent culturel, étant 
donné que les haïdouks " gagnaient la forêt » pour fuir les Turcs et, plusieurs siècles plus tard, les partisans s'y rassemblaient pour organiser la lutte contre les nazis. De plus, on se perd facilement dans la forêt.

Les scènes de la bataille d'Aléria sont pleines de références à Napoléon. Le « sommeil d'Osterlix » (AC, 38) est en fait allusion au « soleil d'Austerlitz», le soleil qui s'est levé à l'aube du 2 décembre 1805, jour de la victoire de Napoléon Bonaparte sur les Austro-Russes à Austerlitz. C'est également une allusion à la paresse proverbiale des Corses. Dans sa traduction, Dimitrijević ne transpose que la notion de paresse corse, parce que « la sieste est sacrée chez nous » (D, 37). Klajn, par contre, garde le nom Austerliks qui, étant corse " n'aime pas se lever tôt », mais Klajn remplace la référence au soleil d'Austerlitz par une autre référence culturelle française - citoyen - appellation utilisée pendant la Révolution française, la période terminée justement par le coup d'État de Napoléon en 1799 : ispavan građanin 'citoyen qui a suffisamment dormi' $(\mathrm{K}, 40)$.

Le commentaire des vieillards qui regardent la bataille, La garde se rend et ne meurt pas (AC, 43), est une allusion à une légende populaire selon laquelle Pierre Cambronne, le commandant de Vieille Garde à Waterloo, aurait répondu à la proposition de se rendre aux Anglais : « La garde meurt mais ne se rend pas !» Dimitrijević omet la référence culturelle : Predaju se... 'Ils se rendent' (D, 42). Klajn la remplace par une référence culturelle serbe : Боље роб него гроб 'Mieux vaut être esclave que mourir' $(\mathrm{K}, 45)$. Il s'agit d'une allusion aux paroles prononcées, dans les manifestations de 1941, par ceux qui s'opposaient au pacte qui liait le Royaume de Yougoslavie avec les puissances de l'Axe : Bolje grob nego rob! 'Mieux vaut mourir qu'être esclave'.

\subsection{Les noms propres}

L'onomastique est une source riche et importante du comique dans tous les albums d'Astérix. Soit transparents (révèlent le caractère, le métier, l'origine, le rôle des personnages), soit allusifs (caricatures de personnages réels), les noms propres représentent un grand défi lancé aux traducteurs. Pour traduire les noms propres d'Astérix en Corse, les traducteurs serbes utilisent le plus souvent le procédé de traduction libre. En adoptant différentes stratégies, les traducteurs serbes proposent différentes traductions des noms propres. Le seul procédé qui leur est commun est celui de transcription, utilisé en général pour la transposition des noms propres réels (César-Cezar-Цезар ; CorseKorzika-Корзика) ou bien des noms des héros éponymes (Astérix-AsteriksАстерикс).

Chez Klajn, on peut repérer une tendance à utiliser des mots et des expressions serbes dont les formes sont adéquates à la création des noms 
romains, gaulois, espagnols etc. Même un peu modifiés, ils sont parfaitement compréhensibles pour le lecteur cible :

Assurancetourix 'assurance tous risques' - Хармоникс, de harmonika 'accordéon'

Babaorum 'baba au rhum'15 - Пијеморум, de pijemo rum 'nous buvons du rhum'

Gazpachoandalus 'gaspacho andalou'16 - Смејумус, toujours employé au vocatif : smeju mu se 'on se moque de lui'

Soupalognon y Crouton 'soupe à l'oignon'17 - Тајез Реозакавез, 'il faut le mettre dans la cage', allusion aux noms espagnols terminés en -ez

Mac Anotérapix 'mécanothérapie'18 - Мек Аникекс, de mekani keks 'biscuit mou'

Petibonum 'petit bonhomme' - Падеминаум, de pade mi na um 'il me vint à l'esprit'

Pour les mêmes raisons, Klajn utilise également des mots empruntés à d'autres langues, ainsi que certains culturèmes :

Petitsuix 'petit-suisse"19 - Несквикс, de Nesquik, une marque de poudre chocolatée produite par Nestlé

Relax 'relax' - Корнфлекс, de kornfleks 'corn flakes'

Beaufix 'beau fixe' ou 'beau-fils' - Брусвиликс, le nom de Bruce Willis

Labeldecadix 'La Belle de Cadix'20 - Мобидикс, de Moby Dick, titre du roman d'Herman Melville

Patologix 'pathologique' - Панегирикс, de panegirik 'panégyrique'

Les noms des héros corses sont en général italianisés dans la version de Klajn :

Carferrix 'car ferry' - Макароникс, de makaroni 'macaronis'

Figatellix 'figatelli' 21 - Шпагетикс, de špageti 'spaghettis'

Le clan de Talassotérapix 'thalassothérapie'22 - клан Ариведерчикс, de l'italien arrivederci

Salamix 'salami'23 - Равиоликс, de ravioli 'ravioli'

\footnotetext{
${ }^{15}$ Un savarin servi imbibé d'un sirop au rhum.

${ }^{16}$ Potage espagnol.

${ }^{17} Y$ signifie et en espagnol.

${ }^{18}$ En médecine, une méthode de rééducation qui permet à l'aide d'appareils de mobiliser les articulations.

${ }^{19}$ Une sorte de fromage

${ }^{20}$ Une opérette française

${ }^{21}$ Saucisses de foie de porc corses.

${ }^{22}$ Usage méthodique des bains de mer.

${ }^{23}$ Saucisson sec italien.
} 
La plus intéressante est la traduction du nom d'Ocatarinetabellatchitchix, référence à la chanson du chanteur corse Tino Rossi, Tchi tchi, qui, dans la version de Klajn devient Ћаобамбиникс, peut-être référence à un film yougoslave de 1988, Ćao, ćao, bambina! À plusieurs reprises, le nom du héros corse est mal prononcé. Klajn utilise systématiquement le procédé d'italianisation, en proposant différentes variantes de noms créés à partir de salutations italiennes: Omarinella... - Буонасерасињориникс, de buonasera signorina; Ocatarinetabellaploumploum - Адиобамбиникс, de adio bambino; Ocatarinetabellatsointsoin - Буонђорнобамбиникс, de buongiorno bambino.

Dimitrijević, par contre, transpose le nom d'Ocatarinetabellatchitchix (et ses variantes citées ci-dessus) par le nom de Napoleoniks, allusion à l'origine corse de Napoléon, bien connue dans la culture cible. Cela permet au traducteur d'omettre certains culturèmes corses pour pouvoir les remplacer par les culturèmes monténégrins sans naturaliser excessivement le texte. Le nom de Napoleoniks renvoie toujours à la Corse.

Tandis que Klajn s'occupe, parait-il, de l'aspect linguistique des noms propres, Dimitrijević met en relief le contexte et la fonction des noms propres dans le texte. Les toponymes sont en général transcrits : Babaorum, Laudanum, Akvarijum, Petibonum etc. Certains noms se réfèrent au métier des personnages qui les portent: Assurancetourix devient Tamburiks, de tambura 'tamboura'24 ; Panoramix devient Aspiriniks, d'aspirin 'aspirine' ; Patologix devient Travariks, de travar 'herboriste'.

Dans la traduction de Dimitrijević, la plupart des anthroponymes présentent le caractère des personnages (ce qui n'est pas toujours justifié par leur nom français ou bien par leur fonction dans Astérix) :

Mac Anotérapix - Mak Skrtiks, de škrt 'avare', un stéréotype des Écossais

Relax - Dremiks, de dremati 'faire la sieste'

Chengelédix 'changer les disques' - Lupetaliks, de lupetati 'raconter n'importe quoi'

Sciencinfus - Mudrolijus, de mudar 'sage, prudent'

Dans la traduction de Dimitrijević il y a des noms qui sont soit complètement omis (Gazpachoandalus, Zebigbos, Osterlix ${ }^{25}$ ), soit remplacés par un nom commun ou par une périphrase (Parlomba - ej ženo ${ }^{26}$, Désiderata - mala Oloviksova ${ }^{27}$, Errata - štrkljasta k'o motka ${ }^{28}$ ).

Le procédé le plus intéressant dans cette version serbe est la traduction multiple, qui souligne le mieux l'importance du contexte dans la stratégie de

\footnotetext{
${ }^{24}$ Instrument à cordes des pays balkaniques.

${ }^{25}$ Klajn: Смејумус(е), Бигбос, Аустерликс.

${ }^{26}$ Klajn: Еспадрила.

${ }^{27}$ Klajn: Дискета.

${ }^{28}$ Klajn: Сонатина.
} 
Dimitrijević. Le mot de passe pour la réunion secrète des chefs de clan corses est une onomatopée qui imite des sons que produisent les sangliers (AC, 36 ; $\mathrm{D}, 35)$. Les noms serbes des chefs proviennent donc du domaine lexical des animaux :

Tropolix 'trop poli' - Čekinjiks, de čekinje 'les soies'

Hérettix 'hérétique' - Papakiks, de papak 'sabot des paridigités'

Minéralogix 'minéralogique' - Kopitiks, de kopito 'sabot des imparidigités'

Appatix 'apathique' - Blejoviks, de blejati 'bêler'

Sinfonix 'symphonique' - Njakaliks, de njakati 'braire'

Plaindetix 'plein de tiques' - Mukoviks, de mukati 'mugir'

Plus tard, pendant la bataille, les " grognards » se présentent en tant que guerriers (AC, 43 ; D, 42) : Tropolix devient Čeliks, de čelik 'acier' ; Hérettix devient Drmadžiks, de drmadžija 'celui qui secoue ; Minéralogix devient Barutiks, de barut 'poudre'; Appatix devient Ubojiks, de ubiti 'tuer'; Sinfonix devient Zastaviks, de zastava, zastavnik 'drapeau, porte-drapeau'; Plaindetix devient Izeliks, de izelica 'glouton', parce qu'il mord le centurion.

De même, le nom du préteur Suelburnus, omis à une reprise (D, 33), est traduit par Lezilebijus, de lezilebović 'personne paresseuse' ( $\mathrm{D}, 6,23,26,30)$, pour souligner son caractère, mais aussi par Drparijus, de drpiti 'voler' (D, 14), pour souligner son comportement envers les Corses.

\section{Conclusion}

Dans sa version d'Astérix en Corse, Dimitrijević opte pour la stratégie de naturalisation. Par la suite, les procédés qui y dominent sont ceux de la traduction libre, de la substitution et de la surtraduction. La naturalisation se manifeste surtout par l'emploi des vernaculaires et du langage familier de la culture cible. Selon Berman, il y a toujours une superposition de langues dans une œuvre, " des dialectes coexistent avec une koinê, plusieurs koinai coexistent »(BERMAN 1999 : 66). Cette superposition des langues est menacée par la traduction, car le « rapport de tension et d'intégration existant dans l'original entre le vernaculaire et la koinê, la langue sous-jacente et la langue de surface, etc., tend à s'effacer » (BERMAN 1999 : 66). Puisque, «seules les koinai, les langues 'cultivées', peuvent s'entretraduire », Berman interdit la traduction d'un vernaculaire étranger par un vernaculaire local, ainsi que d'en rajouter, parce que cela « n'aboutit qu'à ridiculiser l'original » (BERMAN 1999 : 64). Pourtant, dans Astérix en Corse ce n'est pas le cas. Il ne s'agit pas d'un texte « sérieux » mais d'un texte dont le but, le skopos, ainsi que celui de sa traduction, est d'engendrer le comique. Les vernaculaires, souvent 
présentés en tant que stéréotypes, sont l'une des plus riches sources d'humour linguistique. En recourant aux culturèmes de la culture cible, à ses dialectes, à son langage familier, Dimitrijević intensifie, accentue, ajoute. Ces mots, « tirés du 'fond' de la langue » (BERMAN 1999 : 94), plus corporels, plus iconiques que la koinê (BERMAN 1999: 64), peuvent transposer l'expressivité du texte source. Puisque toute « bonne » traduction doit toujours abuser (DERRIDA 1998 : 81), Dimitrijević abuse, mais il n'exagère pas. Il s'agit plutôt d'une accentuation sobre (BERMAN 1999 : 95). Le traducteur serbe a trouvé des moyens pour ne pas détruire ce lien fragile entre le texte source et la culture de laquelle il provient : Astérix est toujours un Gaulois qui a visité la Corse. La traduction de Dimitrijević ne trahit jamais l'original ; elle met plutôt en relief le conflit qui est la vie de l'œuvre (BERMAN 1999 : 96), en lui rajoutant cette « nouvelle nouveauté » qu'exige Goethe (BERMAN 1999 : 76), en lui assurant sa continuité vitale (Fortleben) que préconise Benjamin (2016:20).

Klajn, pour sa part, adopte la stratégie d'exotisation, en utilisant principalement les procédés de traduction littérale, d'emprunt direct et de transcription. Il s'efforce de préserver les traits caractéristiques du texte source (images, styles, culturèmes), en essayant de « recevoir l'Autre en tant qu'Autre »(BERMAN 1999: 74). Le seul domaine qui a été traité différemment est celui des noms propres. En les traduisant Klajn utilise toujours la stratégie d'exotisation (italianisation) mais elle produit un effet inverse : elle naturalise le texte, puisque la culture italienne est mieux connue dans la culture cible. De toute façon, Klajn se soucie plus de la forme, de l'aspect linguistique de la bande dessinée. Il n'utilise jamais le procédé d'omission. Il traduit tout, étant fidèle à « la lettre » du texte, à cette littéralité charnelle (BERMAN 1999 : 77), formée des mots, du sens et de l'énergie de l'œuvre qui représentent la manifestation du «monde » de l'œuvre dans sa totalité (BERMAN 1999: 77).

Même s'il propose, à maintes reprises, des sous-traductions, Klajn, tout comme Dimitrijević d'ailleurs, réussit tout de même à transposer ce qu'il y a d'essentiel dans Astérix. En fait, les points forts et les points faibles des traductions serbes ne se révèlent que si l'on compare ces dernières non pas à l'original, mais l'une à l'autre. Séparées, les deux traductions, tout à fait différentes, l'une poétique et l'autre philologique, fonctionnent parfaitement dans le polysystème cible, transmettant le comique d'Astérix, à la fois particulier et universel, de sorte que la bande dessinée, dans toute sa verboiconicité, continue à vivre dans un autre espace linguo-culturel, en faisant rire un autre public. 


\section{Bibliographie}

BALLARD 2005 : BALLARD, Michel. « La Lecture Des Designateurs de Referents Culturels ». Revista Babilónia. 2005. <http://revistas.ulusofona. pt/index.php/babilonia/article/viewFile/1721/1377>. 20.10.2019.

BENJAMIN 2016 : BENJAMIN, Valter. Zadatak prevodioca. Iskustvo $i$ siromaštvo, prev. Jovica Aćin. Beograd: Službeni glasnik, 2016, 17-40. [orig.] БЕНЈАМИН, Валтер. Задатак преводиоца. Искуство и сиромаштво, прев. Јовица Аћин. Београд: Службени гласник, 2016, 17-40.

BERMAN 1999 : BERMAN, Antoine. La traduction et la lettre ou l'auberge du lointain. Paris : Éditions du Seuil, 1999.

CHEVALIER, GHEERBRANT 1982 : CHEVALIER, Jean et Alain GHEERBRANT. Dictionnaires des symboles. Paris : Robert Laffont/ Jupiter, 1982.

CROS 2011 : CROS, Bernard. « Du village d'Astérix au village global : historique de cinquante ans de succès ". Le tour du monde d'Astérix. 2011. <https://books.openedition.org/psn/6991?lang=en>. 15.10.2019.

DERRIDA 1998 : DERRIDA, Jacques. « Retrait de la métaphore ». Psyché. Inventions de l'autre t. 1 (1998) : 63-93.

ĐURIN, JOVANOVIĆ 2019 : ĐURIN, Tatjana et Ivan JOVANOVIĆ. «An Intangible but very Loud Heritage: Swear Words in Serbian. » Revista de etnografie şi folklor $\mathrm{n}^{\circ} 1-2 / 2019$ (2019) : 94-115.

FARINA 2011 : FARINA, Annick. « Les « Realia francophones » dans les dictionnaires : le modèle d'une traduction exotisante ». ÉLA. Études de linguistique appliquée $\mathrm{n}^{\circ} 164$ (2011) : 465-477.

FLORIN 1993 : FLORIN, Sider. « Realia in translation ». In : Zlateva, Palma. (éd.). Translation as Social Action. Russian and Bulgarian Perspectives (1933): 122-128. London: Routledge.

HENRY 2003 : HENRY, Jacqueline. La traduction des jeux de mots. Paris : Presses Sorbonne Nouvelle, 2003.

KAUFMANN 1998 : KAUFMANN, Judith. «Astérix : les jeux de l'humour et du temps ». Ethnologie française XXVIII-3 (1998) : 327-337.

MAGUET 1998 : MAGUET, Frédéric. «Astérix, un mythe ? Mythogénèse et amplification d'un stéréotype culturel ». Ethnologie française XXVIII-3 (1998) : 317-326.

MEYER 2011 : MEYER, Jean-Paul. «Formes et enjeux de la traduction interculturelle : l'appropriation des stéréotypes nationaux dans quatre traductions des Aventures d'Astérix ». Le tour du monde d'Astérix. 2011. $<$ https://books.openedition.org/psn/7012>. 15.10.2019.

NIÉGOCH 1917 : PÉTROVITCH-NIÉGOCH, Pierre II. Les Lauriers de la montagne, traduit par Divna Vékovitch. Paris : Berger-Levrault, 1917, v. 1133-1134. 
NJEGOŠ 1959 : NJEGOŠ, P. Petar. Gorski vijenac. Beograd : Rad, 1959.

PALMA 2006: PALMA, Silvia. « La traducción de los elementos culturales : el caso de Astérix y Mafalda ». In : Bruña Cuevas, M. et al. (dir.). La cultura del otro: español en Francia, francés en España : 900-909. Sevilla: Universidad de Sevilla, 2006.

POPOVIĆ, NIKOLIĆ 2017 : POPOVIĆ, Nataša et Jelena NIKOLIĆ. « La traduction des références culturelles dans les bandes dessinées ». Godišnjak Filozofskog fakulteta u Novom Sadu XLI-3 (2017) : 357-378.

RMS 2007 : Rečnik srpskoga jezika. Novi Sad: Matica srpska, 2007. [orig.] Речник српскога језика. Нови Сад: Матица српска, 2007.

ROLLO 2017 : ROLLO, Alessandra. «Aspects linguistiques et idéologoculturels dans la traduction de l'humour. Le cas de la bande dessinée Agrippine. » MonTI. Monografías de traducción e interpretación $\mathrm{n}^{\circ} 9$, (2017) : 181-218.

STEVANOVIĆ 2010 : STEVANOVIĆ, Dragoljub. « Dorćolac na privremenom radu u Zemunu ». Politika. 18.09.2010. <http://www. politika.rs/sr/clanak/149507/Dorcolac-na-privremenom-radu-uZemunu>. 20.10.2019.

TLFi 2004 : Le Trésor de la Langue Française informatisé. Disponible sur http://atilf.atilf.fr/tlf.htm

TOUILLIER-FEYRABEND 1998 : TOUILLIER-FEYRABEND, Henriette. « Une traduction du comique est-elle possible ? Compte rendu de la table ronde des journées d'étude de la Société d'Ethnologie française consacrées à L'univers d'Astérix (Paris, 24-25 février 1997) ». Ethnologie française XXVIII-3 (1998) : 375-377.

VIRGILE : VIRGILE, P. Maro. Georgicon. <http://www.thelatinlibrary.com/ vergil/geo2.shtml>. 20.10.2019.

\section{Sources}

AC : Goscinny, René et Albert Uderzo. Astérix en Corse. Paris : Hachette, 2011.

D : Gošini i Uderzo. Asteriks na Korzici, prevod : Đorđe Dimitrijević. Novi Sad : Forum - Marketprint, 1987.

K: Gošini i Uderzo. Asteriks na Korzici : dvadeseta Asteriksova pustolovina. Beograd : Politikin zabavnik, 1997. [orig.] ГОШИНИ и УДЕРЗО. Астерикс на Корзищи: двадесета Астериксова пустоловина. Београд: Политикин забавник, 1997. 
Татјана Ч. Ђурин

Наташа Н. Поповић

\section{ДВА КОНАЧИШТА ЗА ДАЛЕКОГ: ДВА СРПСКА ПРЕВОДА СТРИПА АСТЕРИКС НА КОРЗИЦИ}

У раду се анализирају два српска превода стрипа Астерикс на Корзищи и преводилачки поступци које су користили Ђорђе Димитријевић и Иван Клајн. Посебна пажња је посвећена превођењу хумора, са циљем да се утврди да ли преводиоци успевају да очувају комични ефекат изворног текста. Традуктолошка анализа, заснована на теоријским разматрањима Антоана Бермана, подразумева упоредно проучавање изворног текста и превода и обухвата различите изворе хумора, као што су: игре речи, разговорни језик, културне референце и сл. Преводиоци користе различите преводилачке стратегије (Димитријевић се служи стратегијом подомаћивања, док се Клајн опредељује за потуђивање), али упркос томе успевају да очувају хумор изворног текста.

Кључне речи: превођење, стрип, Астерикс на Корзици, хумор, француски језик, српски језик 